\title{
Impact of Corona Virus Disease 2019 pandemic on adherence to gluten-free diet in Indian patients with celiac disease
}

\author{
Wajiha Mehtab ${ }^{1,2} \cdot$ Ashish Chauhan $^{1} \cdot$ Ashish Agarwal ${ }^{1} \cdot$ Alka Singh $^{1} \cdot$ Mahendra Singh Rajput $^{1} \cdot$ Srikant Mohta $^{1}$. \\ Vikas Jindal $^{1} \cdot$ Vikas Banyal $^{1}$ - Anam Ahmed ${ }^{1}$ - Atreyi Pramanik ${ }^{1} \cdot$ Nikhil Vij $^{3} \cdot$ Ashu Miyan $^{1} \cdot$ Namrata Singh $^{1}$. \\ Anita Malhotra ${ }^{4} \cdot$ Govind K. Makharia ${ }^{1}$ (i)
}

Received: 24 April 2021 / Accepted: 16 June 2021 / Published online: 7 December 2021

(C) Indian Society of Gastroenterology 2021

\begin{abstract}
Aims Lockdown and restricted mobility due to the pandemic of corona virus disease 2019 (COVID-19) has severely affected the continuity of healthcare of patients with acute and chronic diseases. We evaluated the impact of COVID-19 on the adherence to gluten-free diet (GFD), symptom control, and quality of life (QOL) in patients with celiac disease (CeD).

Methods A questionnaire, consisting of both ad-hoc and validated questions, was created after review of literature, group discussions, and expert meetings. Standardized questionnaires namely CeD adherence test (CDAT), celiac symptom index score, and CeD-related QOL were used. The web-based questionnaire was sent to 3130 patients via social media and 452 responses (14.4\%) were received. Also, additional 68 patients (not available on any social media application) were interviewed telephonically by a trained dietitian.

Results Overall, 505 patients (females: 318 ; mean age: $24.1 \pm 14.2$ years) were included. While only $6.7 \%(n=34)$ had poor compliance to GFD (CDAT > 17) before COVID-19 pandemic, it almost doubled to $12.6 \%(n=64)$ during the COVID-19 pandemic times $(p=0.02)$. Furthermore, $4.9 \%(n=25)$ of patients were diagnosed contacting COVID-19. Interestingly, $73.2 \%$ $(n=370)$ patients preferred online appointment than physical appointment. Most common difficulties faced during lockdown period were high delivery charges for getting gluten-free (GF) food at home (54.4\%), increased prices of regular GF food (43.1\%), and travelling long distance to arrange GF food (44.9\%).

Conclusions The COVID-19 pandemic has substantially affected the adherence, symptom control, and QOL in patients with $\mathrm{CeD}$, attributable to unavailability, shortage of money, and heightened cost of GF food. The pandemic has offered an opportunity to practice teleconsultation approach for patients with $\mathrm{CeD}$.
\end{abstract}

Keywords Adherence $\cdot$ Celiac disease $\cdot$ COVID-19 $\cdot$ Gluten-free diet $\cdot$ Quality of life

\section{Introduction}

The corona virus disease 2019 (COVID-19) pandemic has led to almost global lockdown from March 2020 till

Govind K. Makharia

govindmakharia@gmail.com

1 Department of Gastroenterology and Human Nutrition, All India Institute of Medical Sciences, Ansari Nagar, New Delhi 110 029, India

2 Department of Home Science, University of Delhi, New Delhi 110 001, India

3 Member, Celiac Support Group, New Delhi 110 029, India

4 Department of Food Technology, Lakshmibai College, University of Delhi, New Delhi 110 001, India
June 2020. In order to escalate the facility for patients with COVID-19 and also to avoid crowding in the healthcare facilities, restrictions were imposed for many patients including patients with chronic diseases. Such restrictions have affected continuity of care of patients with chronic diseases, including those with celiac disease (CeD) [1].

Celiac disease, an autoimmune enteropathy, occurs in genetically susceptible individuals and is triggered by a protein called gluten, present in cereals such as wheat, barley, and rye [2]. Lifelong and complete avoidance of gluten in the diet is the only treatment for patients with $\mathrm{CeD}$ at present $[3,4]$. Adherence to gluten-free diet (GFD) is essential for the control of the disease [5]. Availability of gluten-free (GF) food is one of the most 


\section{Bullet points of the study highlights}

\section{What is already known?}

- Lockdown and restricted mobility due to the pandemic of corona virus disease 2019 has severely affected the continuity of healthcare of patients with acute and chronic diseases.

\section{What is new in this study?}

- The pandemic has affected the adherence to gluten-free diet and symptom control in patients with celiac disease due to poor availability, high cost of gluten-free food and high delivery charges.

- Almost two third patients preferred online consultation.

\section{What are the future clinical and research implications of the study findings?}

- Strategies should be made to maintain a gluten-free food supply chain, online consultation and monitoring of these patients in case of such nation-wide or regional lockdowns.

important factors that determine appropriate adherence to GFD [6]. While medical shops and local stores were allowed to remain open to fulfill the general need of citizens, GF food was not frequently available in all these local stores. The production of GF food in many countries including India is still evolving, and not widely available [7]. A large number of patients with CeD manage their disease by using non-wheat-, non-barley-based cereal consumption or procurement of GF flour from stores. Although many certified GF food items are imported from other countries, being expensive [8-11], these are consumed by a small number of patients on a regular basis. With imposition of almost sudden lockdown, many patients could not procure enough GF food essentials. Furthermore, the production, import, and distribution of GF food items were also severely affected.

With such restrictions and availability of GF food, we hypothesized that the compliance to GFD might have been affected in many patients with $\mathrm{CeD}$ that might have resulted in the break in adherence and resultant symptoms. With overall environment of panic and uncertainty, the mental health of citizens and more so of those with chronic diseases has also been affected [12, 13]. We therefore planned to assess the impact of COVID-19 on the adherence to GFD, symptom control, and quality of life (QOL) in Indian patients with CeD using web-based questionnaire. We also wanted to understand the various forms of difficulties faced by patients in maintaining GFD and innovative ways to overcome those barriers by them during the pandemic. The whole purpose of this study was to understand these dynamics so that preventive strategies can be planned during any similar event in future.

\section{Methods}

\section{Development and distribution of questionnaire}

An expert panel consisting of 3 gastroenterologists, 3 dieticians, and 6 individuals with $\mathrm{CeD}$ was assembled to discuss the impact of COVID-19 pandemic on adherence to GFD and the factors affecting the adherence, clinical response to diet (symptom control), and QOL. Over a series of meetings and web meetings, a set of 7 domains relevant to $\mathrm{CeD}$ and GFD were constructed, including the following: (a) adherence to GFD, (b) CeD-related symptoms, (c) QOL, (d) GF food stock/ supply, (e) support, (f) difficulties faced during lockdown. For assessing the adherence, CeD-related symptoms, and QOL, standardized and validated questionnaires namely, CeD adherence test (CDAT), celiac symptom index (CSI), and CeD-related quality of life (CD-QOL), were used, respectively, along with a few ad-hoc questions developed specifically for each of the three domains. CDAT is a 7-item questionnaire, having five responses, with score ranging from 1 to 5 . Scores of $<13$ are associated with good GFD adherence, 1317 with average, and > 17 with poor GFD adherence [14]. Adherence was assessed both before and during the lockdown period. CSI is a 16-item questionnaire, with subscales of "specific symptoms" and "general 
health" consisting of 11 and 5 items, respectively. Each item has five responses, score ranging from 1 to 5 . Scores of $\leq 30$ are associated with both high QOL and excellent GFD adherence, which together are quite suggestive of clinical remission. Conversely, a score of 45 or more is associated with relatively poor QOL and worse GFD adherence suggesting ongoing active $\mathrm{CeD}$ [15]. CD-QOL is a 20-item questionnaire, having four clinically relevant subscales (limitations, dysphoria, health concerns, and inadequate treatment). Each item has five responses, with scores ranging from 1 to 5 . The overall score is expressed on a scale of $20-100$, with a higher score indicating poorer QOL [16].

For the remaining domains, a bank of items was developed in a way that questions were representative of the areas studied. Next sequential meetings and discussions with individuals with $\mathrm{CeD}$ and their family members discussed the domains and items decided on by the expert panel. The final question bank consisted of 78 questions, with 11 questions in demographics section and 67 relating to the domains selected (Supplementary Table 1). The questionnaire was then structured in a web survey (google form), and the link was distributed among 15 patients for pilot testing. It helped in assessing the time required for filling the form, answer choices, any discrepancies in selected domains, difficulties faced by patients in filling the form, and appropriateness of the questions to the target population. Pretesting helped in revising the phrases to be maximally understood by the target patients. The questionnaire was sent in both English and Hindi languages. The web-based questionnaire was then sent via social media application namely Whatsapp in 18 Indian celiac support groups (135-246 members in each group), which consists of patients with $\mathrm{CeD}$ and their family members. Patients who were not available on any social media application were directly called on their phone from the directory of Celiac Clinic of our institution. A trained dietitian interviewed the patients thoroughly and noted down the responses.

\section{Ethical approval}

The study was approved by the Ethics Committee of All India Institute of Medical Sciences, New Delhi (Ref. No. IEC-796/07.08.2020). The identity, contact details, and other personal details of the patients have not been disclosed in the public forum. Participants were asked to fill the questionnaire only if they consented to participate in the study. Thus, submission of the filled questionnaire is a representation in itself that the participants have given their consent.

\section{Statistical analysis}

Data are presented as proportions, median, mean, and standard deviation (SD) as appropriate. Paired $t$-test was used to compare the continuous variables. Univariate logistic regression was used to assess the factors affecting the adherence to GFD, before and during the period of lockdown. A $p$-value of less than 0.05 was considered statistically significant. Statistical analysis was performed using Statistical Package for the Social Sciences (SPSS) v20.0 (SPSS Inc., IBM Corporation, Chicago, Illinois, USA).

\section{Results}

We sent the web-based questionnaire to 3130 patients on Whatsapp (a social media application) and received 452 responses (response rate: $14.4 \%$ ). Among those 452 patients who filled the online survey, 15 responses were rejected because of incomplete responses. We also contacted 68 patients telephonically who were not available on any social media application. Therefore, total number of participants included in this study was $505.81 .1 \%$ of the responders were from northern India (Delhi: 31.1\%; Haryana: 18.8\%; Uttar Pradesh: 16.6\%; Punjab: 14.7\%). The mean age of patients was $24.6 \pm 14.4$ years (females-311, 61.6\%). Ninety-eight (19.4\%) patients with $\mathrm{CeD}$ reported having another member having $\mathrm{CeD}$ in the family, more so among female relatives (mothers $15.3 \%$ and sisters $21.4 \%$ ). Overall, $35.2 \%(n=$ 178) of patients were living in the red zone for COVID-19 (areas or hotspots with the highest caseload of COVID-19 and restricted movement of people in and out of the zone). The socio-demographic details of the participants are presented in Table 1.

\section{COVID-19 in patients with CeD}

Overall, $10.8 \%(n=55)$ of patients reported symptoms of pneumonia or flu-like symptoms including sore throat, fever, running nose, and body pain. However, only $4.9 \%(n=25)$ of them and $10.8 \%(n=55)$ of their family members reported as being diagnosed with COVID-19. Overall, 29.7\% $(n=150)$ of patients considered themselves to be at a greater risk of having COVID-19 because they had CeD.

\section{Effect on the adherence to GFD}

\section{Self-reported adherence}

Overall, 68\% $(n=343)$ patients reported maintaining good adherence to GFD before lockdown. With institution of lockdown or restricted availability, 20.5\% ( $n=104), 38.2 \%(n=$ $193)$, and $41.2 \%(n=208)$ patients reported that their 
Table 1 Socio-demographic profile of patients with celiac disease

\begin{tabular}{lc}
\hline Socio-demographic characteristics & $n(\%)$ \\
\hline Gender & \\
Male & $194(38.4)$ \\
Female & $311(61.6)$ \\
Follow-up for treatment of CeD & \\
At our institution & $152(30.1)$ \\
Being treated elsewhere & $353(69.9)$ \\
Follow-up since diagnosis of CeD & \\
1-5 years & $245(48.5)$ \\
5-10 years & $178(35.2)$ \\
10-15 years & $65(12.9)$ \\
$>15$ years & $17(3.4)$ \\
Family history of CeD & $98(19.4)$ \\
First degree relatives & $6(1.2)$ \\
Father & $15(2.9)$ \\
Mother & $12(2.4)$ \\
Brother & $21(4.1)$ \\
Sister & $44(8.8)$ \\
Other relatives & \\
\hline
\end{tabular}

$C e D$ celiac disease

adherence to GFD got completely affected, partially affected, and not affected at all, respectively. Of the 343 patients who reported maintaining good adherence to GFD before the national lockdown, $52.4 \%(n=180)$ reported decreased compliance with GFD during the lockdown period, with $16.3 \%(n=$ 53) of patients resorting to regular consumption of wheat based diets. Overall, 24\% ( $n=120)$ of patients reported intake of wheat-based food items (source of gluten) during the period of lockdown.

\section{Assessment of adherence based on celiac disease adherence test}

The mean CDAT scores before and during the lockdown due to COVID-19 were $12.08 \pm 3.3$ and $12.37 \pm 3.6$, respectively. Overall, 58.6\% reported good compliance to GFD (CDAT score $<13$ ) before COVID-19 pandemic, which reduced to $52.1 \%$ during the lockdown. While only $6.7 \%$ of patients reported poor compliance to GFD (CDAT score $>17$ ) before COVID-19 pandemic, which almost doubled to $12.6 \%$ during the lockdown period $(p=0.02)$. Further, the maximum poor compliant patients were young adults (age range: 20-40 years), compared to other age groups (Table 2).

\section{Effect of lockdown on symptoms of CeD}

Approximately, 94\% ( $n=472)$ of the patients with CeD reported not observing any new symptom during the period of lockdown. The mean CSI score of the patients during the period of lockdown was $28.3 \pm 10.1$. Overall, $65.3 \%(n=$ $303), 24.7 \%(n=125)$, and $9.9 \%(n=50)$ of patients reported good, average, and poor symptom control, respectively (Table 3). Further, the maximum number of patients having poor symptoms control was young adults (age range: $20-40$ years) (Table 3 ).

\section{Effect of lockdown on the quality of life}

While one-third of the patients $(33.6 \%, n=170)$ were not worried or tensed about the COVID-19 pandemic, $23.9 \%$ ( $n$ $=121), 17.6 \%(n=89), 14.2 \%(n=72)$, and $10.5 \%(n=53)$ were slightly, moderately, quite a bit, and greatly worried, respectively. Among those who reported being greatly worried, $73.5 \%(n=39 / 53)$ were women, mostly $>40$ years of age. In our study, the median CD-QOL score of patients during lockdown was 56 (range: 20-93). Approximately, 45\% of patients, irrespective of the age group, had high CD-QOL scores, depicting their poor QOL during the pandemic.

\section{Support from health care providers}

During the period of lockdown, $45.1 \%(n=227)$ and $54.6 \%$ $(n=276)$ patients did not require the need to consult a physician or a dietician, respectively. Majority of the patients $(73.2 \%, n=370)$ preferred online consultation rather than visiting any hospital or clinic. Nevertheless, when required to have a consultation, while $36.2 \%(n=183)$ of patients were able to connect with a physician or gastroenterologist, $3.6 \%(n=18)$ failed to get an appointment for consultation. Similarly, $12.8 \%(n=65)$ of patients were able to connect with a dietitian/nutritionist, but $9.1 \%(n=46)$ patients could not find any. For whichever query or requirement, only $19.8 \%(n=100)$ of patients were able to reach out to their regular doctor(s) (whom they were consulting before lockdown), while $4.3 \%(n=22)$ of patients did not have any contact information of their respective physicians. Similarly, when required, only $10.1 \%(n=51)$ of patients were able to reach out to their regular dietitian, while $9.3 \%(n=46)$ of patients did not have any contact information available.

\section{Difficulties faced in procuring GF food}

The various difficulties faced by patients in procuring GF food and maintaining GFD during the period of lockdown are listed in Table 4 . The most common difficulties reported by nearly half of the patients were paying higher delivery charges for getting GF food at home (52.4\%), higher prices of regular GF food during lockdown (43.15\%), and travelling long distance to arrange GF food (44.9\%). 
Table 2 Celiac disease adherence test score of patients with celiac disease

\begin{tabular}{|c|c|c|c|c|c|c|}
\hline & \multicolumn{3}{|c|}{ Before COVID-19 pandemic } & \multicolumn{3}{|c|}{ During COVID-19 pandemic } \\
\hline & Good $n(\%)$ & Average $n(\%)$ & Poor $n(\%)$ & Good $n(\%)$ & Average $n(\%)$ & Poor $n(\%)$ \\
\hline \multicolumn{7}{|c|}{ Gender-wise distribution } \\
\hline Males $(n=194)$ & $124(24.5)$ & $55(10.8)$ & $15(2.9)$ & $112(22.1)$ & $60(11.8)$ & $22(4.3)$ \\
\hline Females $(n=311)$ & $172(34.0)$ & $120(23.7)$ & $19(3.7)$ & $151(29.9)$ & $118(23.3)$ & $42(8.3)$ \\
\hline Total $(n=505)$ & $296(58.6)$ & $175(34.6)$ & $34(6.7)$ & $263(52.1)$ & $178(35.2)$ & $64(12.6)$ \\
\hline \multicolumn{7}{|c|}{ Age-wise distribution (in years) } \\
\hline$<12(n=110)$ & $85(16.8)$ & $24(4.7)$ & $1(0.1)$ & $80(15.8)$ & $25(4.9)$ & $5(0.9)$ \\
\hline $12-19(n=126)$ & $73(14.4)$ & $48(9.5)$ & $5(0.9)$ & $75(14.8)$ & $37(7.3)$ & $14(2.7)$ \\
\hline $20-40(n=201)$ & $91(18.0)$ & 84 (16.6) & $26(5.1)$ & $71(14.0)$ & $91(18.0)$ & $39(7.7)$ \\
\hline$>40(n=68)$ & $47(9.3)$ & $19(3.7)$ & $2(0.3)$ & $37(7.3)$ & $25(4.9)$ & $6(1.1)$ \\
\hline
\end{tabular}

CDAT score $\leq 13=$ good, $13-17=$ average, $\geq 17=$ poor. $C D A T$ celiac disease adherence test, COVID-19 corona virus disease 2019

\section{GF food stock/supply}

Almost two-third of patients $(62.5 \%, n=316)$ could not find any GF food in their nearby stores. Only $26.7 \%(n=135)$ of patients reported that GF food was kept as an essential food item in stores nearby their residence, while $10.7 \%(n=54)$ of patients were completely unaware of it because they did not step out to check. In procuring GF food from market or different parts of the city, 28.8\% $(n=145)$ did not face any difficulty in travelling and $52.8 \%(n=267)$ faced some hurdles, while $18.4 \%(n=93)$ could not travel at all. A large proportion of the patients $(58.8 \%, n=297)$ ordered GF food via various websites, food delivery applications, or directly from the manufacturers/whole-sellers. Surprisingly, $12.2 \%$ ( $n$ $=62$ ) of patients were unaware of any such online sources to purchase GF food. The patients who ordered GF food from online sources faced difficulties like delayed delivery, no delivery in certain areas, delivery far away from home, and

Table 3 Celiac symptom index score of patients during the period of lockdown

\begin{tabular}{|c|c|c|c|}
\hline & Good, $n(\%)$ & Average, $n(\%)$ & Poor, $n(\%)$ \\
\hline Total $(n=505)$ & $330(65.3)$ & $125(24.7)$ & $50(9.9)$ \\
\hline \multicolumn{4}{|c|}{ Gender-wise distribution } \\
\hline Males $(n=194)$ & $141(27.9)$ & $32(6.3)$ & $21(4.1)$ \\
\hline Females $(n=311)$ & $189(37.4)$ & $93(5.9)$ & $29(5.7)$ \\
\hline \multicolumn{4}{|c|}{ Age-wise distribution (in years) } \\
\hline$<12(n=110)$ & $84(16.6)$ & $23(4.5)$ & $3(0.5)$ \\
\hline $12-19(n=126)$ & $96(19)$ & $24(4.7)$ & $6(1.1)$ \\
\hline $20-40(=201)$ & $109(21.5)$ & $54(10.6)$ & $38(7.5)$ \\
\hline$>40(n=68)$ & $41(8.1)$ & $24(4.7)$ & $3(0.5)$ \\
\hline
\end{tabular}

CSI score $\leq 30=$ good, $30-45=$ average, $\geq 45-80=$ poor. $C S I$ celiac symptom index heavy delivery charges for getting GF food at home. Nearly, half of the patients $(49.1 \%, n=248)$ received support from fellow patients in knowing shops to purchase GF food.

\section{Factors affecting the adherence to GFD}

On univariate logistic regression, the rate of non-adherence to GFD was high in those having COVID-19 (OR $=8.96$; 95\% CI: $3.6-21.1 ; p=0.001)$ and or anyone in the family (OR = $2.54,95 \%$ CI: $1.4-4.5 ; p=0.02$ ). Among other factors, heavy delivery charges for getting GF food at home, higher prices of regular GF food during lockdown and shortage of money to buy food items played statistically significant role in affecting the adherence of a patient to GFD (Table 5).

\section{Discussion}

The present study has focused on the impact of COVID-19associated countrywide lockdown and restricted mobility on the adherence to GFD, symptom control, QOL, and

Table 4 Difficulties faced by patients with celiac disease in procuring gluten-free food during the period of lockdown

\begin{tabular}{ll}
\hline Difficulties faced in procuring GF food & $n(\%)$ \\
\hline Heavy delivery charges for getting GF food at home & $265(52.4 \%)$ \\
Travelling long distance to procure GF food & $227(44.9 \%)$ \\
Increased prices of regular GF food during lockdown & $218(43.1 \%)$ \\
Shortage of grains to GF flour at home & $141(27.9 \%)$ \\
No transport available to travel to get groceries & $112(22.1 \%)$ \\
Shortage of money to buy food items during lockdown & $105(20.7 \%)$ \\
Disrupted courier services & $25(4.9 \%)$ \\
\hline
\end{tabular}

$G F$ gluten-free 
Table 5 Factors affecting adherence to gluten free diet during the period of lockdown COVID-19 corona virus disease 2019, $C e D$ celiac disease, $G F$ gluten-free

\begin{tabular}{lll}
\hline Variables & $\begin{array}{l}\text { Odds ratio (OR) } \\
95 \% \text { confidence interval }\end{array}$ & $p$-value \\
\hline Female gender & $1.3(0.8-2.1)$ & 0.19 \\
Occurrence of COVID-19 in CeD patients & $8.9(3.6-21$. & 0.001 \\
Occurrence of COVID-19 in the family member & $2.5(1.4-4.5)$ & 0.02 \\
Having symptoms of pneumonia/sore throat & $1.9(1.04-3.5)$ & 0.03 \\
Heavy delivery charges for getting GF food at home & $2.1(0.9-2.7)$ & 0.81 \\
Travelled long distance to arrange GF food & $1.1(0.7-1.6)$ & 0.62 \\
Non-availability of the transport to get groceries & $0.9(0.5-1.6)$ & 0.91 \\
Shortage of GF flour at home & $1.09(0.6-1.7)$ & 0.70 \\
Disrupted courier services & $0.3(0.09-1.07)$ & 0.03 \\
Higher prices of regular GF food during lockdown & $1.8(0.9-2.2)$ & 0.08 \\
Shortage of money to buy food items during lockdown & $1.9(0.9-2.6)$ & 0.05 \\
\hline
\end{tabular}

difficulties faced by Indian patients with $\mathrm{CeD}$. The lockdown state affected the adherence to GFD in almost $60 \%$ of Indian patients (both children and adults) with $\mathrm{CeD}$. The safety measures to prevent and control the spread of COVID-19 had a profound impact on the GF food supply to patients with CeD. Overall, $10.8 \%(n=55)$ of patients reported symptoms of pneumonia or flu-like symptoms; however, only $4.9 \%$ of them and $10.8 \%$ of their family members were diagnosed to have COVID-19. However, this cannot be generalized to the entire CeD population as many may have remained asymptomatic and not get tested. The non-adherence to GFD was almost 8fold higher in patients with $\mathrm{CeD}$ who had developed COVID19 compared to those who did not.

Overall, one-third (29.7\%) of patients considered themselves at greater risk of having COVID-19 because they had CeD. Similar results have been shown in Italy where almost $20 \%$ of patients felt more vulnerable to COVID-19 because they had CeD [17], although Emmi et al. reported that the risk of severe acute respiratory syndrome corona virus-2 (SARS-CoV-2) infection was similar among people with autoimmune disorders and the general population [18]. Moreover, in our study, only $5 \%$ of patients with $\mathrm{CeD}$ got infected with SARS-CoV-2 virus. Further, almost $11 \%$ of patients in our study faced flu-like symptoms including sore throat, fever, running nose, and bodypain. Yet another Italian study showed similar results where $13.7 \%$ of patients showed flu-like symptoms with no diagnosis of COVID-19 [19].

Atleast $10.5 \%(n=53)$ of patients reported being greatly worried about the COVID-19 pandemic, majority being women $(73.5 \% ; n=39 / 53)$ over $>40$ years of age. Further, $45 \%$ of patients, irrespective of the age group, had high CD-QOL scores, depicting their poor QOL during the pandemic. However, the QOL may have been affected by the overall situation of panic during the pandemic, and not just due to CeD-related factors.
In the present study, although a greater number of patients were able to reach out to the respective physician or dietitian for online consultation, a few could not. The appropriate adherence to GFD is the key to the success of treatment, and maintenance of adherence requires repeated consultation with a dietitian or a nutritionist. It is obvious that limited availability of GF food created by this pandemic must have raised questions in the mind of patients and their families about the GF nature of the available food and how best to maintain adherence in such situation. It thus looks most appropriate that necessary information about maintaining adherence is provided to patients through health care providers and patient support groups. This pandemic provided and established the value of teleconsultation as an important mode of consultation with the physicians and dieticians for management of diseases like CeD. Our findings are in line with the Italian study (2020) where patients also preferred remote consultation during the period of lockdown [17].

The adherence of a patient to GFD was significantly affected by factors such as high prices of GF food, high delivery charges, and shortage of money to buy food items during the lockdown state. Majority (62\%) of the patients could not find GF food items in their nearby stores. Nonavailability of GF food has already been established as one of the major barriers in adherence to GFD by Indian patients with CeD [20], which got accentuated in pandemic. In our study, more than $50 \%$ of those patients who maintained strict adherence to GFD before the pandemic, reported difficulties in maintaining their adherence to GFD during the pandemic. India is still in its infancy in terms of production of GF food items, and the one's mostly produced are from small-scale or middle-scale industry [7]. The shutdown of factories, shortage of labor, and limited imported GF food items contributed to further increase in the prices of GF food during lockdown. The most 
devastating impact of COVID-19 and the subsequent lockdown had been on the economically backward classes, with limited access to proper healthcare and other resources.

While the individual rate of adherence to GFD was significantly affected, the mean CDAT scores, however, remained almost similar during the lockdown compared to before the lockdown $(12.08 \pm 3.3$ before vs. $12.37 \pm$ 3.6 during lockdown). CDAT has been proven in multiple patient cohorts to adequately represent the adherence to GFD; however, CDAT has its own pitfalls. CDAT has components on QOL, symptoms, and gluten adherence. There are questions like "how many times you have eaten outside" which due to lockdown has resulted in a zero response to this question. Interestingly, even in patients having good adherence before the lockdown, the adherence scores got further better (decreased from 12 towards 7) because of lack of travel or use of outside food.

During such nation-wide or regional lockdown or curfew states, methods should be devised to make availability in the local stores of foods items required for patients with special food needs. Naturally GF grains like millets should be made available in all grocery stores and ration shops at subsidized rates for such patients. All patients should be provided the contact details of their concerned healthcare provider so that teleconsultations by doctors and dietitians can be accomplished. All patients should have access to celiac support groups to gather information about GFD.

The strength of the present study is the use of standardized questionnaires assessing different domains. The questions were kept both open-ended and multiple choice answers in order to not influence the answers of the patients. Although the questionnaire was not validated by any celiac society, majority of its components have been validated and used in $\mathrm{CeD}$. One of the major limitations of this study is the use of social media platforms where response rate is generally limited. Since only $14.4 \%$ of patients with $\mathrm{CeD}$ responded to the questionnaire, this data may not be representative of the whole population. Furthermore, since the assessment of adherence to GFD before COVID-19 pandemic has been based on recall, the CDAT scores for that period could have been affected by optimistic recalls. While social media platforms allow reaching out to a larger number of subjects/patients, the reliability of such response may not be perfect.

In conclusion, the COVID-19 pandemic has substantially affected the adherence, symptom control, and QOL in patients with $\mathrm{CeD}$, attributable to unavailability, shortage of money, and heightened cost of GF food. The pandemic has offered a great opportunity to practice teleconsultation approach for CeD healthcare.
Supplementary Information The online version contains supplementary material available at https://doi.org/10.1007/s12664-021-01213-4.

Acknowledgements We acknowledge the support of all the patient support groups for helping us relay this questionnaire and all the patients with celiac disease participating in this study.

Author contribution Wajiha Mehtab: review of literature, development of questionnaire, data collection, data analysis, drafting of manuscript; Ashish Chauhan: questionnaire development, review of literature, critical review of the paper; Ashish Agarwal: critical review of the paper; Mahendra Singh Rajput: data collection, critical review of the paper; Alka Singh: critical review of the paper; Srikant Mohta: data collection, critical review of the paper; Vikas Jindal: data collection, critical review of the paper; Vikas Baniyal: data collection, critical review of the paper; Anam Ahmed : critical review of the paper; Atreyi Pramanik: data collection, critical review of the paper; Nikhil Vij: questionnaire development, data collection; Ashu Miyan: data collection; Namrata Singh: study supervision, critical review of the paper; Anita Malhotra: critical review of the paper; Govind K. Makharia: overall guarantor of the paper, designed the study concept, supervised the study, data analysis, and finalization of the paper. All authors revised and approved the final version.

\section{Declarations}

Competing interests $\mathrm{WM}, \mathrm{AC}, \mathrm{AA}, \mathrm{AS}, \mathrm{MSR}, \mathrm{SM}, \mathrm{VJ}, \mathrm{VB}, \mathrm{AA}, \mathrm{AP}$, $\mathrm{NV}, \mathrm{AM}, \mathrm{NS}, \mathrm{AM}$, and GKM declare no competing interests.

\section{Guarantor of the article Dr. Govind K Makharia.}

Ethics statement The study was performed conforming to the Helsinki declaration of 1975, as revised in 2000 and 2008 concerning human and animal rights, and the authors followed the policy concerning informed consent as shown on Springer.com.

Disclaimer The authors are solely responsible for the data and the contents of the paper. In no way, the Honorary Editor-in-Chief, Editorial Board Members, the Indian Society of Gastroenterology or the printer/ publishers are responsible for the results/findings and content of this article.

\section{References}

1. Palmer K, Monaco A, Kivipelto M, et al. The potential long-term impact of the COVID-19 outbreak on patients with noncommunicable diseases in Europe: consequences for healthy ageing. Aging Clin Exp Res. 2020;32:1189-94.

2. Lindfors K, Ciacci C, Kurppa K, et al. Coeliac disease. Nat Rev Dis Primers. 2019;5:3.

3. Dicke WK. Treatment of celiac disease. Dutch J Med. 1951;95:124-30.

4. See J, Murray JA. Gluten-free diet: the medical and nutrition management of celiac disease. Nutr Clin Pract. 2006;21:1-15.

5. See JA, Kaukinen K, Makharia GK, Gibson PR, Murray JA. Practical insights into gluten-free diets. Nat Rev Gastroenterol Hepatol. 2015;12:580-91.

6. Mehtab W, Singh N, Malhotra A, Makharia GK. All that a physician should know about gluten-free diet. Indian J Gastroenterol. 2018;37:392-401.

7. Rajpoot P, Makharia GK. Problems and challenges to adaptation of gluten free diet by Indian patients with celiac disease. Nutrients. 2013;5:4869-79. 
8. Fry L, Madden AM, Fallaize R. An investigation into the nutritional composition and cost of gluten-free versus regular food products in the UK. J Hum Nutr Diet. 2018;31:108-20.

9. Gorgitano MT, Sodano V. Gluten-free products: from dietary necessity to premium price extraction tool. Nutrients. 2019;11:1-12.

10. Singh J, Whelan K. Limited availability and higher cost of glutenfree foods. J Hum Nutr Diet. 2011;24:479-86.

11. Lee AR, Wolf RL, Lebwohl B, Ciaccio EJ, Green PHR. Persistent Economic Burden of the Gluten Free Diet. Nutrients. 2019;11:399.

12. Saqib MAN, Siddiqui S, Qasim M, et al. Effect of COVID-19 lockdown on patients with chronic diseases. Diabetes Metab Syndr. 2020;14:1621-3.

13. Louvardi M, Pelekasis P, Chrousos GP, Darviri C. Mental health in chronic disease patients during the COVID-19 quarantine in Greece. Palliat Support Care. 2020;18:394-9.

14. Leffler DA, Dennis M, George JBE, et al. A simple validated gluten-free diet adherence survey for adults with celiac disease. Clin Gastroenterol Hepatol. 2009;7:530-6.

15. Leffler DA, Dennis M, George JE, et al. A validated diseasespecific symptom index for adults with celiac disease. Clin Gastroenterol Hepatol. 2009;7:1328-34.
16. Dorn SD, Hernandez L, Minaya MT, et al. The development and validation of a new coeliac disease quality of life survey (CDQOL). Aliment Pharmacol Ther. 2010;31:666-75.

17. Siniscalchi M, Zingone F, Savarino EV, D'Odorico A, Ciacci C. COVID-19 pandemic perception in adults with celiac disease: an impulse to implement the use of telemedicine. Dig Liver Dis. 2020;52:1071-5.

18. Emmi G, Bettiol A, Mattioli I, et al. SARS-CoV-2 infection among patients with systemic autoimmune diseases. Autoimmun Rev. 2020;19:1-5.

19. Zingone F, D'Odorico A, Lorenzon G, Marsilio I, Farinati F, Savarino EV. Risk of COVID-19 in celiac disease patients. Autoimmun Rev. 2020;19:102639.

20. Rajpoot P, Sharma A, Harikrishnan S, Baruah BJ, Ahuja V, Makharia GK. Adherence to gluten-free diet and barriers to adherence in patients with celiac disease. Indian J Gastroenterol. 2015;34:380-6.

Publisher's note Springer Nature remains neutral with regard to jurisdictional claims in published maps and institutional affiliations. 